\title{
LOS KALATHOI DE CUELLO ESTRANGULADO DE LAS NECROPOLIS IBERICAS DE COIMBRA DEL BARRANCO ANCHO (JUMILLA, MURCIA)
}

\author{
José Miguel GARCÍA CANO \\ Museo de Murcia
}

\section{Resumen}

En este artículo se estudian los kalathoi de «cuello estrangulado» de las necrópolis ibéricas de Coimbra del Barranco Ancho, donde se han podido diferenciar tres variantes en función de su morfología. Aparecen cronológicamente en el siglo IV antes de Jesucristo, pero tienen un especial auge durante el siglo III y primeros años del siglo II a. de C..

\section{Summary}

This article is a study of a shape of pottery called kalathos with a «strangulationneck» form which comes from iberian necropolis of Coimbra del Barranco Ancho, where there are three different forms depending on their morphology. This shape began in the fourth century b.C., but has a special period of increase during the third century and the first years of the second century before Christ.

El conjunto ibérico de Coimbra está situado en las proximidades de Jumilla, 4.5 kilómetros al sur-sureste de la ciudad, es posiblemente el yacimiento ibérico más relevante de la comarca Jumilla-Yecla. A la enorme superficie que ocupa hay que sumar la existencia de tres necrópolis a su alrededor y contar con un centro de culto propio sólo a unos cientos de metros al este del núcleo principal habitado.

Nuestro trabajo se centrará en los kalathoi de cuello estrangulado aparecidos en las necrópolis excavadas sistemáticamente entre 1980-1987, esto es, de la Senda y del Poblado. Así denominadas por sus respectivas ubicaciones, la primera en el camino que comunica el Poblado con el Santuario y la segunda por encontrarse en la misma planicie que el habitat ( MOLINA GARCIA-MOLINA GRANDE-NORDS- 
TROM, 1976; INIESTA-PAGE-GARCIA CANO, 1987; GARCIA CANO, 1994. Con la bibliografía actualizada).

\section{MORFOLOGIA Y TIPOLOGIA}

El término kalathos es de eminente raigambre clásica, referido a los cestos de mimbre en la antigua sociedad griega. En la actualidad se emplea para definir una forma relativamente rara del repertorio ceramológico ático (RICHTER-MILNE, 1973,14). Es sin embargo, un recipiente paradigmático de la vajilla ibérica con cuerpo cilíndrico y borde plano saliente al exterior, conocido popularmente como sombrero de copa, donde este vocablo ha encontrado su mejor definición. Nosotros hemos optado por él para la variante de cuello «estrangulado» al ser la más frecuente en la bibliografía especializada, entre otros, C. Aranegui y E. Pla (1981,79), F. Presedo (1982,295), M. Pellicer, J.L. Escacena y M. Bendala (1983,95), S. Broncano y J.J.Blanquez $(1985,277)$ y J.M. García Cano $(1987,116)$. No obstante algunos investigadores han elegido distinto apelativo, así E. Cuadrado $(1972,129)$ y C. Mata $(1991,77)$ los denominan «tarro» o tarro cilíndrico, M.J. Conde $(1992,109)$ prefiere el de urna kalathoide, Solveig Nordström los definió como sítulas sin asa ( NORDSTROM, 1969-73,176).

Son siete los vasos a analizar aparecidos en otras tantas incineraciones. Uno pertenece a la necrópolis de la Senda, n. ${ }^{\circ}$ S-642 (T.3S) y el resto a la del Poblado tumbas $n .{ }^{\circ} 2,19,43,51,67$ y $68^{\prime}$. La forma se caracteriza por tener cuerpo cilíndrico o ligeramente troncocónico, borde exvasado levemente colgante y fondo plano o cóncavo con umbo en el centro. Es el estrechamiento o «estrangulamiento» que configura el cuello lo que los diferencia, junto con el borde, de los sombreros de copa. Dentro de la clasificación de la cerámica fina de Coimbra ocupan la forma 12 (GARCIA CANO, 1994,4120). Se han podido diferenciar tres modelos en función de su morfología:

Variante 1.- Borde ligeramente colgante con carena muy marcada en el inicio del cuello, cuerpo cilíndrico, fondo cóncavo y umbilicado. n.. S-642 (T.3S); 1846 (T.19); 3494 (T.43); 5732 (T.68) (figura 3).

Variante 2.- Borde colgante o apuntado al exterior. El cuerpo se va estrechando desde el hombro hasta la base. La carena que marca la reducción del cuello muy suavizada. Fondo plano o ligeramente cóncavo. n. 4069 (T.51) y 5724 (T.67) (figura 3).

Variante 3.- Borde al exterior. El cuerpo se estrecha casi imperceptiblemente desde el hombro, fuertemente señalado, a la base. Entre la carena y el labio

I Para diferenciar los objetos de ambas necrópolis, se ha optado por colocar la letra «S» de Senda a todas las referencias de materiales o tumbas de dicha necrópolis. 
del borde se define un cuello cilíndrico bastante amplio. Fondo totalmente plano. n. 190 (T.2) (figura 3).

En Coimbra suponen el $6 \%$ de la cerámica ibérica de los ajuares funerarios, reduciéndose al $3,8 \%$ al considerar toda la cerámica indígena y sólo un $2,4 \%$ del total cerámico.

Su uso doméstico es desconocido, pero E. Cuadrado supone que debieron emplearse para contener semisólidos como miel o manteca (CUADRADO, 1972,149), aunque los ejemplares de mayor tamaño también pudieron guardar cereal u otros sólidos. En el mundo funerario, cinco de las piezas -tumbas n. ${ }^{2} 19,43,51,67$ y 68 han sido utilizadas como urnas cinerarias, mientras que el n. ${ }^{\circ}$ S-642 de la tumba 3 de la necrópolis de la Senda, de la que no sabemos su función entre el ajuar, aunque J. Molina lo define como «urna» (MOLINA-MOLINA-NORDSTROM, 1976,77). Por tanto sólo el n. ${ }^{\circ} 190$ (T.2), único representante de la variante 3, formaba parte del ajuar funerario como vaso de ofrenda.

Los kalathoi de cuello estrangulado más comunes en nuestras necrópolis son los de la variante 1, esto es, con cuerpo cilíndrico y carena marcada. En este grupo se enmarca el ejemplar de la Senda que además, es el que tiene una cronología más elevada de la variante y de la forma entre el 375-350 a.C.. Los restantes cubren aproximadamente todo el siglo III a.C. Así el n.․5732 (T.68) se sitúa entre el 275-225 a.C. y los números 1848 y 3494 (T.19 y T.43) corresponden al último periodo de funcionamiento de la necrópolis con dataciones de finales de la centuria y primeros años del siglo II a.C. (GARCIA CANO, 1994,3138-3139).

Las dos unidades de la variante 2 , es decir, con el cuerpo que se estrecha progresivamente desde el hombro hasta la base se datan en la segunda mitad del siglo III a.C. El n.. 4069 (T.51) tiene una amplitud cronológica que cubre los segundos cincuenta años del siglo III a.C., mientras que el n. 5724 (T.67), pieza de reducidas dimensiones, debe ubicarse entre el cambio de siglo y los primeros años de la segunda centuria anterior a Cristo (GARCIA CANO, 1994,3138-3139).

El kalathoi de la variante 3, n. ${ }^{\circ} 190$ (T.2), se fecha a mediados de la segunda mitad del siglo IV a.C. (375-325 a.C.) (GARCIA CANO, 1994, 3137).

Estos recipientes están muy extendidos por toda el área ibérica desde Levante y Sureste hasta Andalucía, siendo un vaso relativamente común. Por esta razón renunciaremos a señalar paralelos formales para incidir más en aquellos conjuntos que proporcionen cronología precisa que permita determinar el origen y la perduración del tipo.

Corresponde a la forma 12 de la tipología de la cerámica fina del Cigarralejo, donde E. Cuadrado ha diferenciado cuatro variantes en función de detalles morfológicos del cuello y boca, siendo la cuarta variante (F12d) un tarro de pequeñas dimensiones, casi una botellita (CUADRADO, 1972,129, tabla IX). Este es uno de los 
yacimientos con más kalathoi de cuello estrangulado publicados -31 piezas- que se fechan a lo largo del siglo IV a.C.,excepto los n.o 1690 (T.184) que presenta una amplitud cronológica del siglo III a.C. y el n. 2956 (T.290) datable en el siglo II a.C. sin poder hacer mayores precisiones en estos dos kalathoi (CUADRADO - QUESADA, 1989,58, figura 13).

Se identifica con la forma 16 de la tipología de la cerámica ibérica de Aranegui y Pla en donde también se han diferenciado cuatro subvariantes con relación a la disposición del labio del borde vuelto/moldurado y a la configuración del perfil abombado/tendencia cilíndrica (ARANEGUI - PLA, 1981,79). Nuestros ejemplares de la variante 1 se relacionan con 1a F16c y los de la segunda con F16a. Carmen Aranegui y Enrique Pla señalan que los kalathoi de cuello estrangulado aparecen en el País Valenciano en el siglo IV a.C. , seguramente en la segunda mitad, y que en el siglo III a.C. son sustituidos por kalathoi tradicionales mediante ejemplares de transición (ARANEGUI - PLA, 1981,78). Contamos con otros dos estudios monográficos sobre yacimientos de la Comunidad Valenciana, Federico Rubio clasificó los de la necrópolis de la Albufereta (Alicante) en dos variantes : «A» para los sombreros de copa, «B» para los de cuello estrangulado (RUBIO GOMIS, 1986,350-354) y Consuelo Mata, que en un reciente trabajo sobre el poblado de los Villares (Valencia) los ha incluído como "tarros cilíndricos» dentro de su Grupo II, tipol0 (MATA,1991,77) documentándose en el nivel Villares IV que corresponde culturalmente a la fase ibérica plena que fecha la investigadora entre el segundo cuarto del siglo $\mathrm{V}$ a.C. y finales del siglo III a.C. (MATA, 1991,193-195).

En Andalucía han sido clasificados por J. Pereira quien los integra en su Grupo formal $8 \mathrm{~B}$ y $\mathrm{C}$, con ejemplares en yacimientos de Andalucía Oriental y Occidental. La variante $\mathrm{B}$, hombro con fuerte carena, tendría sus prototipos más antiguos en la transición de los siglos V-IV antes de Cristo, en ambos extremos de la cuenca del Guadalquivir que sin embargo se limitan al sector oriental de la cuenca a partir del siglo IV. Por lo que respecta a la variante $\mathrm{C}$, sin carena en el hombro, sería una forma típica del siglo IV a. C. documentada también en la parte oriental de la cuenca (PEREIRA SIESO, 1988,160, figura 10 y 11).

Del mismo modo la forma ha sido catalogada en la necrópolis de Baza (Granada) donde es bastante abundante -29 items.- con una cronología del siglo IV a. C.. F. Presedo ha diferenciado siete variantes (PRESEDO, 1982,295). Por último mencionar que se integra en el Grupo II, tipo I en sus variantes A y B de la tipología establecida por D. Vaquerizo para la necrópolis de Almedinilla (Córdoba) con amplia cronología (VAQUERIZO, 1988-89,120-121).

\section{CRONOLOGIA Y CONSIDERACIONES FINALES}

Los kalathoi de cuello estrangulado más antiguos proceden de Andalucía, así la urna tipo VII de la necrópolis de la Bobadilla (Jaén) se fecha entre la segunda mitad 
del siglo VI y mediados del siglo siguiente que es la cronología global que otorgan sus excavadores al yacimiento (MALUQUER - PICAZO - RINCON, 1973,5). No obstante los caracteres de este kalathos difieren un tanto del modelo clásico: labio del borde recto y cuello bien definido a partir de la carena lo que tampoco es común. Los restantes parámetros si se corresponden con los kalathoi de cuello estrangulado: Paredes cilíndricas, base casi plana o ligeramente cóncava en el centro, tamaño, etc. (MALUQUER - PICAZO - RINCON, 1973,34-39, figura 23). Pensamos que la cronología de esta necrópolis podría prolongarse quizás hasta finales del siglo $\mathrm{V}$ antes de Jesucristo. También de la segunda mitad del siglo $\mathrm{V}$ tenemos una pieza n.. 1328 del nivel 14 del Cerro Macareno (Sevilla) (PELLICER - ESCACENA - BENDALA, 1983,95, figura 46-1328).

Dentro del sureste y levante los vasos con cronología más elevada, tránsito de los siglos V-IV, serían los procedentes de la tumba n. 549 del Cabecico del Tesoro (GARCIA CANO, 1987) y el de la incineración n. ${ }^{\circ} 14$ de la necrópolis de la Hoya de Santa Ana (Albacete) (BLANQUEZ, 1990,294-299) que puede fecharse en estos mismos años por la presencia de un askos de cerámica ática de figuras rojas y dos pateritas de barniz negro -F21/25AL.- entre el ajuar (TRIAS, 1967,422-423, lámina CLXXXVI-1 y 3 a 5). Datables a lo largo del siglo IV a. C. estarían los vasos del Cigarralejo, Baza y otros yacimientos andaluces y levantinos. Citaremos el sombrero de copa con cuello estrangulado del punto 54 de la necrópolis de Cabezo Lucero (Alicante) (ARANEGUI, 1992,34, n. ${ }^{\circ}$ 34) cuya cronología entre el 375-350 es muy similar al de nuestro enterramiento más antiguo con kalathoi -tumba 3S-. Esta pieza viene a confirmar la existencia del modelo con cuello estrangulado en el País Valenciano con cronología de la primera mitad del siglo IV antes de Jesucristo.

La cronología más tardía para los kalathoi de cuello estrangulado lo aportan yacimientos como el Amarejo (Albacete), donde un nutrido grupo de estos vasos están en uso en el último nivel de habitación durante la segunda mitad avanzada del siglo III a. C. (BRONCANO - BLANQUEZ, 1985,241-243, n.o 263-267, figuras 134135). En este momento o ya en los primeros años del siglo II a.C. hay que datar el vaso procedente de la tumba L-81 de la necrópolis de la Albufereta (RUBIO GOMIS, 1986,201-202, figura 90).

Como consideración final puede afirmarse que el kalathos de cuello estrangulado está plenamente configurado como forma hacia finales del siglo V a. C. -Cerro Macareno o la Bobadilla- siendo su etapa de mayor difusión y popularidad el siglo IV, tanto en Andalucía -Baza, Almedinilla, Toya o Galera- como en el área de Murcia -Cigarralejo, Cabecico del Tesoro- y sureste en general -Albufereta o Cabezo Lucero-. En estas fechas es cuando se introduce el tipo en Coimbra, ejemplar de la tumba $3 \mathrm{~S}$ de la Senda. La forma sin embargo no se hace familiar en Jumilla hasta la segunda mitad de la centuria siguiente con cinco sobre seis vasos de la necrópolis del Poblado usados como urnas cinerarias. Estos recipientes siguen en boga en la necró- 
polis hasta el último periodo de funcionamiento del cementerio, n. 1846 y 3494 (tumbas 19 y 43) datables entre finales del siglo y los primeros años del siglo II antes de Cristo, como sucede con los vasos del cercano poblado del Amarejo (BRONCANO - BLANQUEZ, 1985,277-278). En esta fase los kalathoi de cuello estrangulado han desaparecido de una buena parte de los yacimientos aunque aisladamente la forma ha podido perdurar hasta el siglo II a. C. como en la tumba 290 del Cigarralejo datable globalmente a lo largo de esta centuria (CUADRADO, 1987,499, figura 217).

\section{$* * *$}

Existen dos fragmentos de kalathoi en la necrópolis del Poblado que por similitud formal deben analizarse junto a los kalathoi de cuello estrangulado. Uno apareció en el ajuar de la tumba 6, n. ${ }^{\circ} 704$, figura 3 . Sólo conserva parte del cuerpo y borde. este es moldurado con sección de cabeza de anade. Tiene decoración geométrica estandarizada en el exterior. Del segundo vaso, n. ${ }^{\circ} 2715$, figura 3 , se ha recuperado un trozo de pared, pero puede adscribirse a los sombreros de copa típicos con borde plano saliente. Se halló dentro del contexto funerario, de la deposición n. 35 que estaba alterada. En el exterior se decora con motivos florales de estilo Elche-Archena.

El kalathos con borde moldurado corresponde a la forma 17a de Aranegui y Pla (1981:79) y al tipo 1 Grupo C-1 de M." José Conde (1990b,152). Esta investigadora piensa que su centro de fabricación debió de estar ubicado en las cercanias de Líria debido a la concentración de hallazgos producidos en los alrededores de la ciudad de Valencia (CONDE BERDOS, 1990b,152, figura 8. Para todos los materiales de Líria véase BONET 1995, 411-412).

La cronología establecida para este tipo de kalathoi puede centrarse en el siglo III a. de C., mayoritariamente hacia su segunda mitad y primeras décadas del siglo II en base a los hallazgos bien documentados de Puntal dels Llops, Monravana, Tossal de San Miguel de Líria, Amarejo y Cabecico del Tesoro. Sólo un ejemplar de la Alcudia se fecharía hacia la primera mitad de la centuria (CONDE BERDOS, 1990b,152-153).

Nuestro kalathos aunque incompleto puede adscribirse al modelo de vaso anteriormente descrito. La tumba $\mathrm{n}^{2} 6$ pertenece a la última fase de uso de la necrópolis y por estratigrafía vertical podría fecharse entre el 250-200 a.C. (GARCIA CANO, 1994,3138). Se integra perfectamente en Ia cronología propuesta para el tipo en función de otros yacimientos. El kalathos seguramente fué utilizado, la tumba estaba alterada, como urna cineraria de la incineración.

El fragmento de pared, n.․ 2715, es de un «sombrero de copa». Está decorado en el exterior con una gran hoja de hiedra muy estilizada que lo relaciona directamente con el estilo Elche-Archena (MENENDEZ, 1988). 
El kalathos como vasija prototípica y paradigmática de la cerámica ibérica está presente en numerosas tipologías de las que señalaremos únicamente las más importantes: Tipo IV de L. Pericot ( 1979,79); forma 13 de la cerámica fina del Cigarralejo (CUADRADO, 1972, tabla X); forma 17b de Aranegui y Pla (1981,79); Grupo II, tipo 7 de Mata $(1991,75)$. M. a José Conde les ha dedicado un estudio en profundidad (CONDE BERDOS, 1990a) y ha incluido nuestro tipo en el Grupo D-2 (CONDE BERDOS, 1990b, 154). Ultimamente J. Pereira al estudiar la cerámica ibérica de la cuenca del Guadalquivir ha insertado los kalathoi dentro del Grupo 8-D (PEREIRA SIESO, 1988,160).

En Murcia dos de los cuatro ejemplares publicados del Cigarralejo se fechan a lo largo del siglo III a.C. -tumbas 90 y319-y los otros en la centuria siguiente -tumbas 198 y 240-. Pudiéndose concretar el n. 1770 (T.198) entre el 125-100 a.C. (CUADRADO Y QUESADA, 1989; 87 figura 14). En Coimbra el único kalathos hallado hasta la fecha en el poblado procede de la habitación I, n. ${ }^{\circ}$ E-S-255/428 (PAGE Y OTROS, 1987; 25). Este vaso estaba en uso en la fase final del habitat del poblado, es decir, entre finales del siglo III a.C. y los primeros decenios de la centuria siguiente. Otra pieza se ha localizado en Bolbax (LILLO CARPIO, 1981;251)

Por lo que respecta al motivo decorativo mediante hojas de hiedra estilizadas, forma parte importante de un buen número de kalathoi. Contamos entre otros de varios items en la necrópolis del Cabecico del Tesoro con paralelos muy próximos en vasos de las necrópolis de la Hoya de Santa Ana (Albacete), Corral de Saus y Cerro Lucena (Enguera). Estos materiales pueden fecharse claramente en el siglo II a.C., tal vez en su segundo cuarto en función del resto de los ajuares funerarios (CONDE BERDOS, 1990;159).

Nuestro fragmento procede de una incineración de la última fase de uso de la necrópolis, ligeramente alterada, pero que puede fecharse por restos de una copa de cerámica campaniense $\mathrm{A}$, hacia los primeros años del siglo II antes de Jesucristo (GARCIA CANO, 1994,3080). Según M. ${ }^{\mathrm{a}}$ José Conde todos estos vasos pudieron fabricarse en un mismo taller enclavado muy probablemente en los alrededores del Verdolay (CONDE BERDOS, 1990b,159).

\section{BIBLIOGRAFIA}

ARANEGUI GASCO, C. (1992): «Cerámica ibérica», Catálogo de la exposición Cabezo Lucero necrópolis ibérica, Guardamar del Segura (Alicante). Alicante, pp. 34-37.

ARANEGUI GASCO, C. Y E. PLA (1981): «La cerámica ibérica». Mesa redonda la baja época de la cultura ibérica. Madrid, pp. 73-144. 
BLANQUEZ PEREZ, J. J. (1990): La formación del mundo ibérico en el sureste de la meseta. (Estudio arqueológico de las necrópolis ibéricas de la provincia de Albacete). Albacete

BONET ROSADO, H. (1995): El Tossal de Sant Miquel de Llíria. La antigua Edeta y su territorio. Valencia

BRONCANO, S. Y J. J. BLANQUEZ (1985): El Amarejo (Bonete, Albacete). EAE 139. Madrid

CONDE BERDOS, M. J. (1990a): La produccio ceramica en el mon iberic: el kalathos, analisi y clasificacio. Tesis doctoral. Inédita. Barcelona

CONDE BERDOS, M. J. (1990b): «Los kalathoi "sombrero de copa" de la necrópolis del Cabecico del Tesoro de Verdolay (Murcia)». Verdolay 2. Homenaje a E. Cuadrado. Murcia, pp. 149-160

CONDE BERDOS, M. J. (1992): Colección arqueológica. Durán/Vall-llosera. Arte ibérico. Barcelona

CUADRADO DIAZ, E. (1972): «Tipología de la cerámica ibérica fina de "El Cigarralejo" Mula. Murcia». Trabajos de Prehistoria 29. Madrid, pp. 125-187

CUADRADO DIAZ, E. (1987): La necrópolis ibérica de "El Cigarralejo". Mula. Murcia. BPH XXIII. Madrid

CUADRADO DIAZ, E. Y F. QUESADA (1989): «La cerámica ibérica de "El Cigarralejo" (Murcia). Estudio de cronología». Verdolay 1. Murcia, pp. 45-115

GARCIA CANO, J. M. (1987): «Una sepultura singular del Cabecico del Tesoro. Verdolay (La Alberca. Murcia)». Anales de Prehistoria y Arqueología 3. Murcia, pp. 115-122

GARCIA CANO, J. M. (1994): Las necrópolis ibéricas en Murcia (Un ejemplo paradigmático: Coimbra del Barranco Ancho. Estudio analítico). Murcia (Microficha)

INIESTA, A., V. PAGE Y J. M. GARCIA CANO (1987): La sepultura setenta de la necrópolis del Poblado. Coimbra del Barranco Ancho (Jumilla). Murcia

LILLO CARPIO, P. (1981): El poblamiento ibérico en Murcia. Murcia

MALUQUER,J., M. PICAZO Y M. A. RINCON (1973): La necrópolis ibérica de la Bobadilla (Jaén). Barcelona

MATA PARREÑO, C. (1991): Los Villares (Caudete de las Fuentes. Valencia). Origen y evolución de la Cultura Ibérica. S. I. P. Serie de Trabajos Varios 88. Valencia

MOLINA, J., M. C. MOLINA Y S. NORDSTROM (1976): Coimbra del Barranco Ancho. Jumilla. Murcia. S.I.P. Serie de Trabajos Varios 52. Valencia

NORDSTROM, S. (1969-1973): La ceramique peinte iberique de la province d'Alicante. Estocolmo 
PAGE, V., J.M. GARCIA CANO, A. INIESTA Y M.J. RUIZ (1987): Coimbra del Barranco Ancho. Diez años de excavaciones. Murcia

PELLICER, M., J. L. ESCACENA Y M. BENDALA (1983): El Cerro Macareno. EAE 124. Madrid

PEREIRA SIESO, J. (1988): «La cerámica ibérica de la cuenca del Guadalquivir I. Propuesta de clasificación». Trabajos de Prehistoria 45. Madrid, pp. 143-173

PERICOT GARCIA, L. (1979): Cerámica ibérica. Barcelona

PRESEDO VELO, F. (1982): La necrópolis ibérica de Baza. EAE 119. Madrid

RICHTER, G. Y M. MILNE (1973): Shapes and names of Athenian vases. Washington (1. ㄹ. Edición 1935)

RUBIO GOMIS, F. (1985): «El yacimiento ibérico del Puig (Alcoy)». Noticiario Arqueológico Hispánico 24. Madrid, pp. 91-157

RUBIO GOMIS, F. (1986): La necrópolis de la Albufereta de Alicante (Valencia. España) Academia de Cultura Valenciana. Serie Arqueológica 11. Valencia

TRIAS, G. (1967): Cerámicas griegas de la Península Ibérica. Valencia

VAQUERIZO GL, D. (1988-89): «Ensayo de sistematización de la cerámica ibérica procedente de las necrópolis de Almedinilla. Córdoba». Lucentum VII-VIII. Alicante, pp. 103-132 


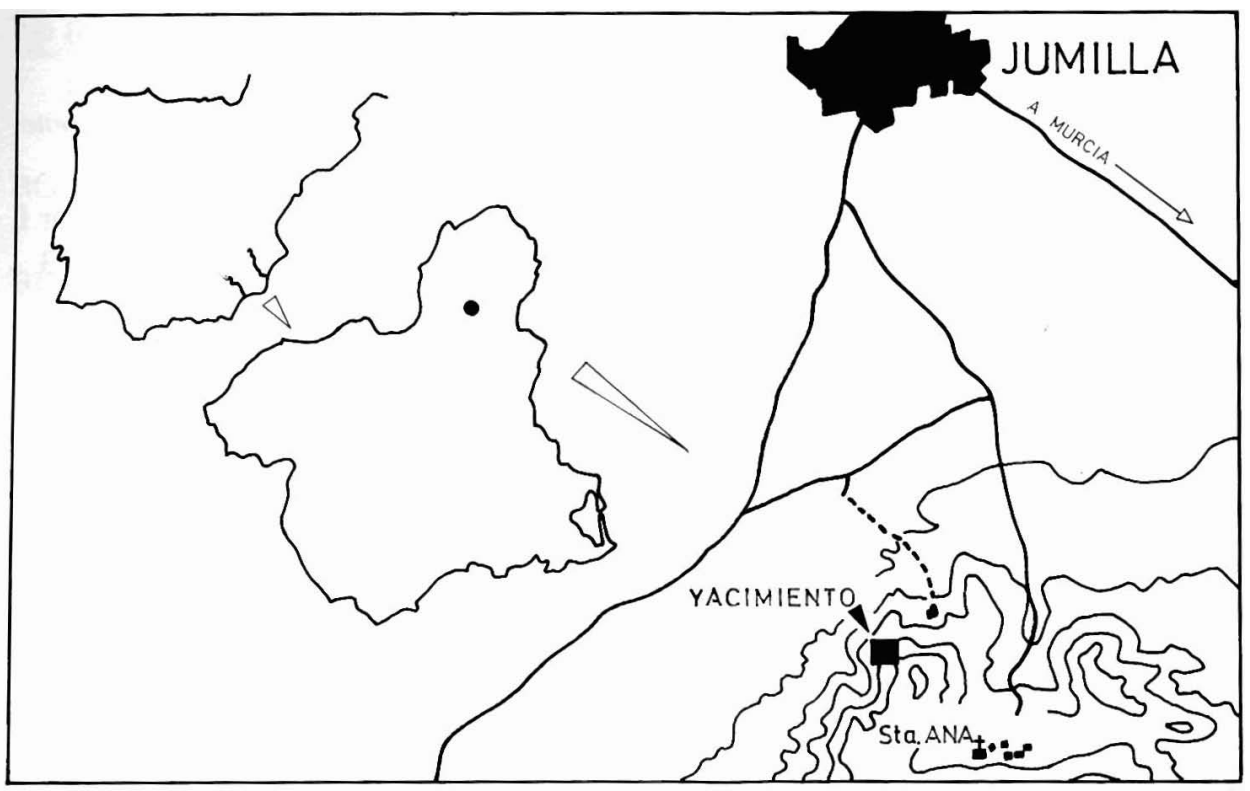

Fig. 1.- Situación del conjunto ibérico de Coimbra del Barranco Ancho (Jumilla).

\begin{tabular}{|c|c|c|c|}
\hline UMBA & NUMERO & 1400 & 350 \\
\hline $3 \mathrm{~S}$ & $S-642$ & & VAR. 1 \\
\hline 2 & 190 & & VAR. 3 \\
\hline 68 & 5732 & & \\
\hline 51 & 4069 & & \\
\hline 6 & 704 & & \\
\hline 67 & 5724 & & \\
\hline 19 & 1846 & & \\
\hline 43 & 3494 & & \\
\hline 35 & 2715 & & \\
\hline
\end{tabular}

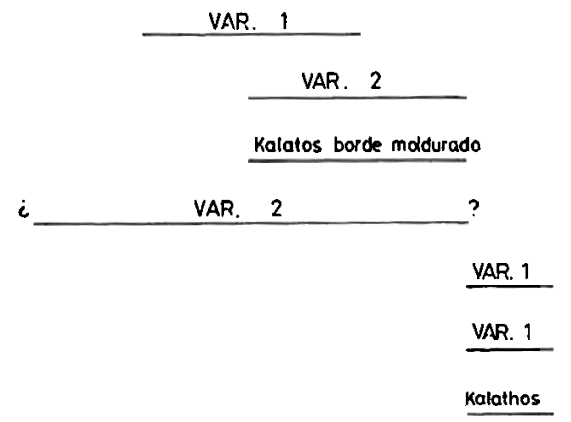

Fig. 2.- Kalathoi de cuello estrangulado (Forma 12 de Coimbra). Cronología. Se incluyen dos piezas por aproximación tipológica: Un fragmento de kalathos con borde ligeramente moldurado n. 704 y un trozo de pared de otro kalathos pensamos que del tipo "sombrero de copa", con decoración floral. Este fragmento es único hasta la fecha en la necrópolis del Poblado, $n .^{\circ} 2715$. 


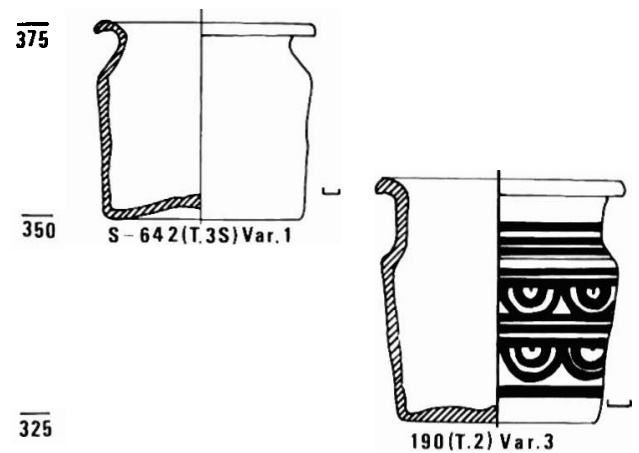

$\overline{300}$
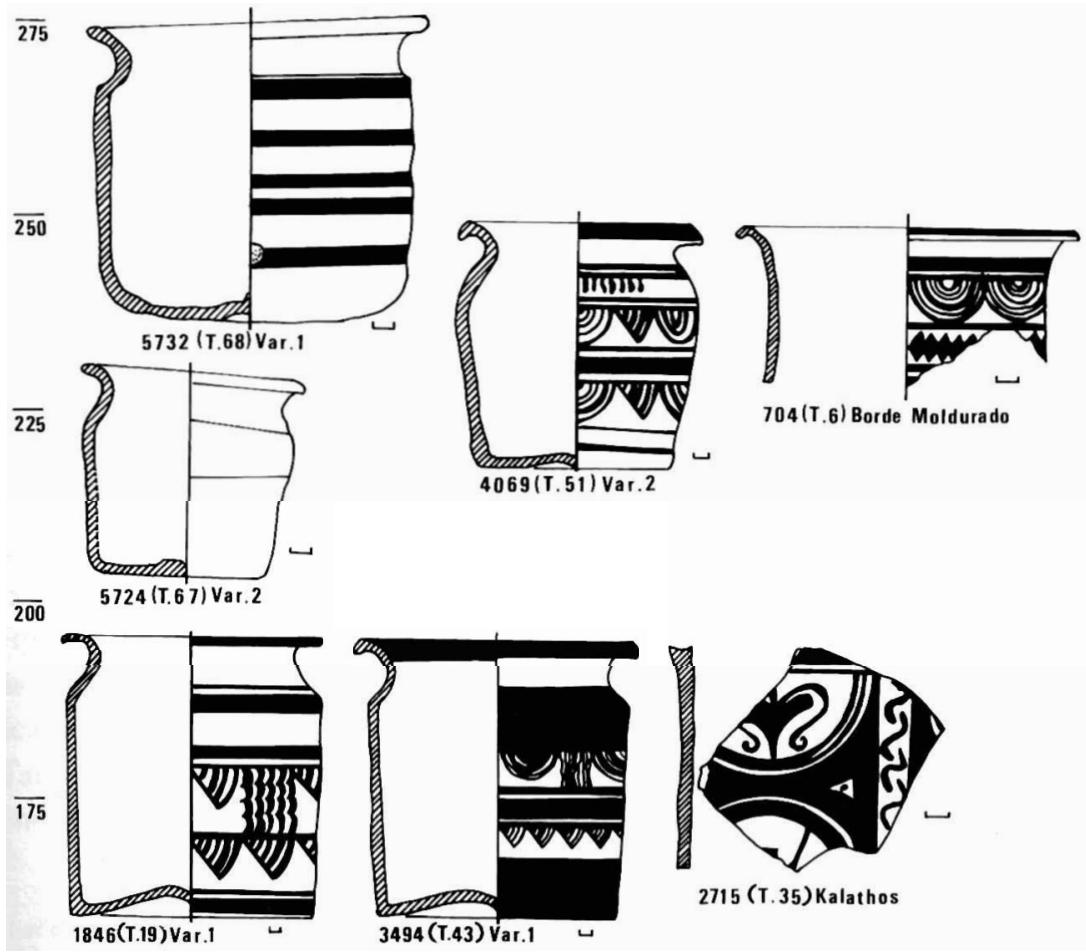

Fig. 3.- Tipologia de los kalathoi de las necrópolis de Coimbra del Barranco Ancho. 